\title{
Análisis de las redes de distribución de balón en fútbol: pases de juego y pases de adaptación
}

\author{
Isidro Maya Jariego \& M. Rocío Bohórquez \\ Universidad de Sevilla, España ${ }^{1}$
}

\section{Resumen}

Este artículo propone distinguir los pases de juego y los pases de adaptación al juego en las aplicaciones del análisis de redes sociales al estudio del fútbol. Las redes de pases de balón permiten describir y evaluar las configuraciones tácticas en el desarrollo de un partido (Bundio y Conde, 2007; Cotta, Mora, Merelo-Molina y Merelo, 2011). El equipo es analizado como una red en la que los jugadores son los nodos y los pases de juego son lazos dirigidos. Los patrones de interacción entre jugadores revelan procesos emergentes que trascienden las estadísticas individuales de corte tradicional. Pese a que las primeras aplicaciones utilizan la matriz bruta de pases de balón, la diferenciación de las relaciones entre jugadores en función del tipo de tarea desarrollada muestra con más claridad los elementos tácticos del juego (Maya Jariego y Bohórquez, 2011). Para ilustrarlo utilizamos datos del Real Betis Balompié en la final de Copa de S. M. El Rey de 2005 y del encuentro de la final de Copa de 2007. Tanto los indicadores de centralidad como las pruebas no paramétricas muestran una clara diferenciación entre la red de pases de juego y la red de pases de adaptación. Los resultados permiten interpretar el desarrollo táctico del partido, que comparamos con la descripción basada en estadísticas individuales de corte tradicional.

Palabras clave: Análisis de redes sociales - Fútbol - Pases de juego - Pases de adaptación al juego.

\begin{abstract}
The network of who passes the ball to whom describes the tactical configurations in the development of a soccer match (Bundio y Conde, 2007; Cotta, Mora, MereloMolina y Merelo, 2011). The team is analyzed as a network, with players as nodes and passes as directed edges. The patterns of interaction between players reveal emergent processes that transcend traditional individual statistics. Although the first applications of network analysis to soccer use the matrix of all the passes during the match, the differentiation of the relationships between players depending on the type of work carried out shows more clearly the tactical elements of the game (Maya Jariego y Bohórquez, 2011). To illustrate this we used data from Real Betis in the 2005 Copa del Rey final, as well as the match in the 2007 final. Both centrality measures and nonparametric tests showed a clear differentiation between the passing network and the adaptation network. The results allow interpreting the tactical development of the match. Finally we compare the network analysis of the match with a description based in individual players statistics.
\end{abstract}

Key words: Social Network Analysis - Soccer - Passing Network - Adaptation Network.

1 Contacto: Isidro Maya Jariego, Departamento de Psicología Social, Universidad de Sevilla, 41018, Sevilla, España. Dirección electrónica: isidromj@us.es 


\section{I ntroducción}

En el análisis de los encuentros de fútbol es habitual encontrar referencias a recuperaciones, pérdidas de balón, centros al área, tiros a puerta y otras acciones individuales desarrolladas por los jugadores durante el partido (Anguera, Blanco, Losada y Hernández-Mendo, 2000; Castellano y Hernández-Mendo, 2002). Del mismo modo, las referencias a los tiempos de posesión o al dominio atacante tratan de dar cuenta del trabajo colectivo desarrollado por cada equipo.

Este uso tradicional de las estadísticas individuales - centradas en aspectos técnicos y finalistas- presenta limitaciones cuando pretendemos evaluar el componente táctico del deporte (Chicote, Morante y Vaquera, 2009), un elemento eminentemente colectivo. El comportamiento de los jugadores en el campo depende de relaciones de cooperación-oposición, de forma que la acción individual viene en parte determinada por las sucesivas configuraciones tácticas adoptadas por el conjunto de jugadores en interacción (Lago y Anguera, 2003).

Una propuesta con la que examinar la configuración táctica del equipo ha consistido en la observación conductual de las interacciones, combinando el análisis del comportamiento con elementos propios de la sociometría tradicional (Santoyo, 1994). Las aplicaciones de dicha metodología se han dirigido con frecuencia a mostrar la relación entre la cohesión del grupo y el rendimiento del equipo (Klein y Christiansen, 1969). Siguiendo ese esquema original, con equipos de baloncesto se ha utilizado el análisis secuencial, junto con indicadores sociométricos, en el diseño de mapas socio-conductuales (Hernández-Mendo, González, Ortega, Ortega y Rondan, 1999; Hernández-Mendo y Canto, 2003; Sautu, Garay y HernándezMendo, 2009).

Los mapas socio-conductuales también se han empleado con equipos de fútbol. Castellano, Hernández-Mendo y Haro (2002) analizaron los siete partidos disputados por la selección francesa en el Mundial de Fútbol de Francia '98. El estudio descriptivo muestra cierta relación positiva entre los indicadores de cohesión y relación en las secuencias de pase de balón y el rendimiento colectivo. También se sugiere que dicha estructura de interacción se ve influida por aspectos socio-afectivos y de clima grupal.

Una segunda línea de trabajo se basa en herramientas del análisis de redes sociales (Wasserman y Faust, 1994), que permiten examinar dinámicas de colaboración e intercambio (véase por ejemplo, Peñaranda-Ortega, Quiñones-Vidal \& Osca-Lluch, 2009). Las primeras aplicaciones de este enfoque estructural en contextos deportivos tienen lugar en la década de 1970. En el caso del fútbol, destaca el 
análisis de la final de la Copa Inglesa de 1977 entre el Liverpool y el Manchester United (Gould y Gatrell, 1979). En dicho estudio se evalúa el solapamiento entre subconjuntos y la conectividad de la red. Gould y Gatrell (1979) proponen que "un equipo es más que la suma de sus jugadores individuales y por tanto implica una estructura de relaciones entre el conjunto" (p. 253). Veamos algunos de los desarrollos que han seguido esa idea en el mundo del fútbol, antes de hacer, en ese mismo marco, nuestra propuesta específica de análisis.

\section{Las redes de pases de balón: el caso del Real Betis Balompié en la final de Copa de 2005}

El patrón de juego en un partido de fútbol puede formularse como una red de pases de balón. Los jugadores son nodos y los pases son vínculos orientados, conformando un dígrafo del que su grosor indicará la cantidad de pases entre dos jugadores. Esta forma de reducir la estructura del juego permite examinar el papel de actores individuales $y$, al mismo tiempo, representa el desempeño del equipo en su conjunto. La dinámica del juego se representa en una estructura social de interacción.

Este enfoque proporciona indicadores de trabajo colectivo como la centralidad de grado (el número de pases dados y recibidos), la intermediación (el grado en que un jugador hace de nexo en los caminos más cortos entre otros dos) o la cercanía (cuando un jugador accede o resulta accesible para otros en su equipo) y ubica el rendimiento individual en el contexto de los intercambios del grupo. El pase de balón es un elemento clave del juego puesto que, además de mantener la posesión y evitar que la tenga el contrario, contribuye a la consecución del objetivo, marcar goles. Por lo demás, la distribución de la pelota refleja el estilo de juego y es un elemento de confrontación táctica con el otro equipo.

El uso predominante del análisis de redes ha consistido en estrategias de carácter descriptivo y exploratorio. El cálculo de indicadores de centralidad y la representación de grafos permite describir patrones de juego y explorar elementos tácticos (Bundio y Conde, 2007). También se ha incorporado en las prácticas del entrenamiento deportivo. Por ejemplo, ARSFútbol lo ha aplicado en Argentina en el asesoramiento técnico con equipos de fútbol base. La visualización de la estructura de pases facilita la comunicación de la disposición táctica, mientras que el análisis de centralidad resulta útil en la evaluación del rendimiento individual y colectivo.

Sin embargo, también se ha analizado cómo la estructura de la red de pases influye en el rendimiento del equipo. Concretamente se han evaluado las consecuencias de 
la densidad de la red, la centralidad de jugadores y la existencia de agrupaciones (Bundio y Conde, 2007), el papel de los intermediarios en las secuencias de pases de balón, la densidad entre defensores y atacantes (ARSFútbol, 2011), la centralización de la red (Lee, Borgatti, Molina y Merelo, 2005), e incluso cómo evoluciona la densidad y el coeficiente de aglomeración a lo largo del partido (Cotta, Mora, Merelo-Molina y Merelo, 2011).

Pese a tratarse de una trayectoria de investigación empírica relativamente corta, se han identificado algunos indicadores estructurales de la efectividad en el juego. Por ejemplo, una red con menor centralización que el equipo contendiente, de modo que la generación de pases esté distribuida entre varios jugadores, resulta una ventaja competitiva (Lee et al., 2005). También es positivo un índice de aglomeración comparativamente elevado, conformando grupos de jugadores que se asocian entre sí (Cotta et al., 2011). Del mismo modo, la emergencia de varias tríadas fuertes en el equipo parece más resistente a la interceptación de pases (ARSFútbol, 2011; Cotta et al., 2011). Por su parte, las prácticas defensivas se relacionan con un mejor rendimiento cuando resultan en espacios en la geometría del otro equipo (Gould y Gatrell, 1979).

En todos los casos se utiliza como referencia la matriz bruta de pases de balón a lo largo del partido. Sin embargo, dar a todos los pases el mismo valor, sin atender a las circunstancias del juego, puede enmascarar algunas características relevantes en la configuración táctica del equipo. Por eso, se propone una sencilla innovación en el tratamiento de los datos, consistente en separar los pases de adaptación de los pases de juego (Maya Jariego y Bohórquez, 2011). Como se explicará más adelante, el uso de dos matrices diferentes sirve para distinguir, por poner un ejemplo característico, los pases atrás ante la presión de un contrario de las acciones de juego efectivo. Esta precaución en el procesamiento de los datos mejora el potencial descriptivo de la red de pases, distinguiendo el papel que tienen en la dinámica del juego. Para ilustrarlo, se expone un ejemplo exploratorio con la red de pases del Real Betis Balompié en la final de la Copa de S. M. el Rey de 2005 contra el Club Atlético Osasuna.

Si se toma el conjunto de pases de balón que tuvo lugar a lo largo del partido, obtenemos una representación global del juego (Figura 1$)^{2}$. El análisis de la red permite observar que la distribución del balón está sesgada hacia la mitad derecha

\footnotetext{
${ }^{2}$ En la visualización y edición de los gráficos del Real Betis Balompié han participado los técnicos de ARSFútbol: <http://arsfutbol.wordpress.com>. El tamaño de los nodos indica la cantidad de pases dados y recibidos. Los lazos indican los pases entre compañeros (la intensidad es la fortaleza de la relación).
} 
del equipo. La densidad de pases es claramente superior en esa parte del campo de juego. Se trata de una descripción genérica del flujo de actividad. Atendiendo a los indicadores de centralidad individuales, podemos destacar el papel de Luis Fernández, Assunçao y Fernando en la articulación del conjunto.

En segundo lugar, las cadenas de pases de balón más frecuentes arrojan como resultado el eje del equipo: Juanito-Arzu-Assunçao-Joaquín-Oliveira. Se trata de actores que están conectados por 5 o más pases a lo largo del partido. Para completar el análisis descriptivo, podemos señalar que Luis Fernández es el punto de apoyo preferente de Assunçao en el juego de balón hacia atrás. Curiosamente, cuando entra en juego Varela en sustitución de Arzu (en el minuto 69), se establece una conexión habitual entre Melli y Varela en la subida del balón.

Figura 1. Red total de pases realizados por el Real Betis Balompié.

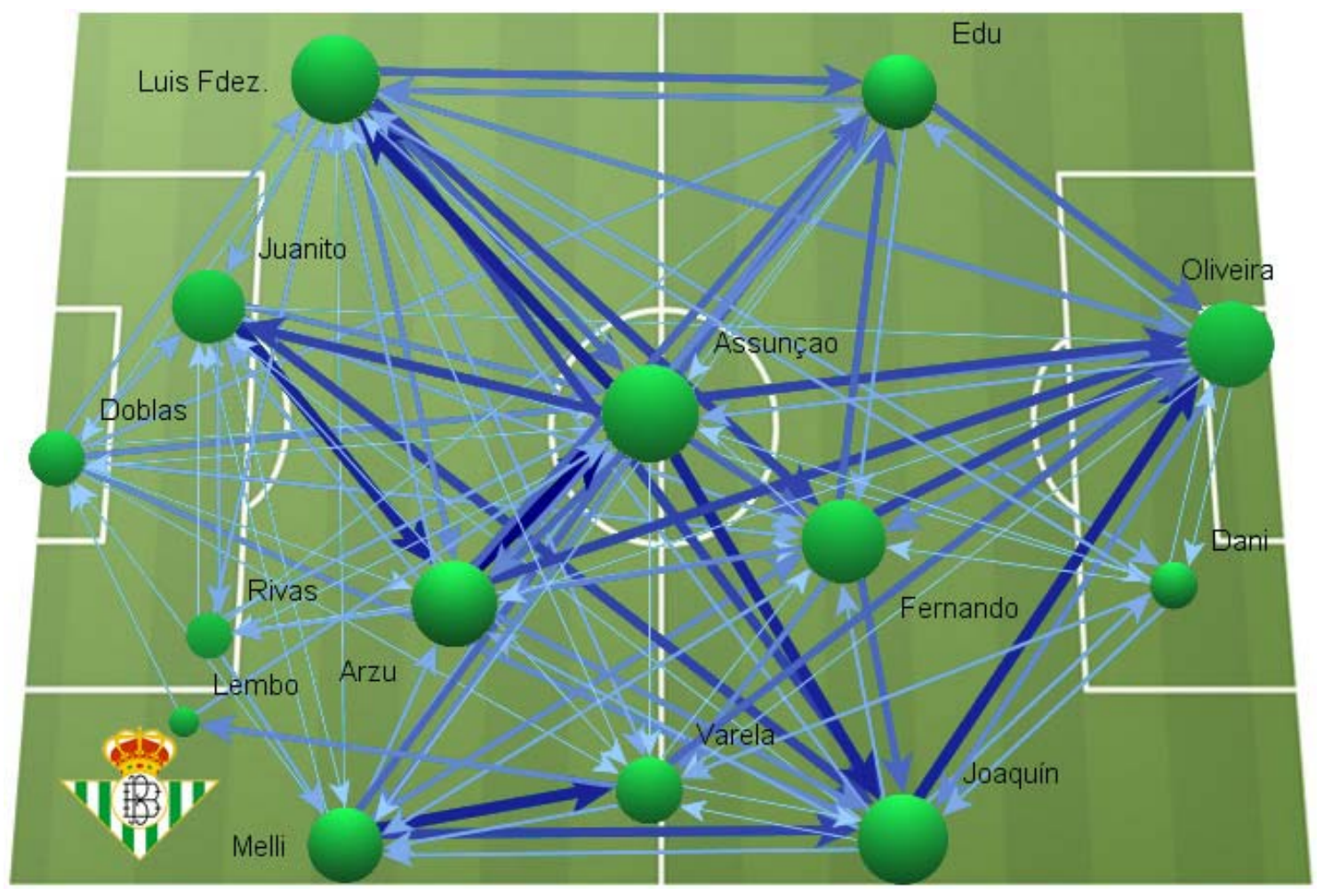

Dividamos a continuación la matriz de pases en dos subconjuntos independientes y mutuamente excluyentes: los pases de juego y los pases de adaptación. Un pase de juego es aquel que implica la transmisión de balón entre dos jugadores del mismo equipo que pretende explotar una ventaja sobre el oponente, ya sea en fase defensiva u ofensiva del juego: centros o incursiones al área, asistencias de gol, paredes, pases a un jugador en mejor situación defensiva/ofensiva o saques de portería en largo, por ejemplo. Por su parte, los pases de adaptación al juego son aquellos que suponen la transmisión de balón entre dos jugadores del mismo 
equipo y que pretende mitigar la presión y/o generar movimiento en el equipo contrario para facilitar una mejor situación defensiva u ofensiva. Los pases horizontales sin presión sobre la posesión, los saques en corto del portero o pases hacia zonas libres de marca serían ejemplos de acciones de adaptación al juego (Galeote, 2003; Lacuesta Salazar, 1997; Moreno, 1998).

Ahora podemos identificar dos estructuras diferenciadas. En la matriz de pases de adaptación son comparativamente más frecuentes los pases hacia atrás. Se trata de intercambios para mantener la pelota, evitar que sea interceptada por los contrarios o marcar el tiempo en diferentes fases del juego. En esta matriz intervienen hasta 10 jugadores (Figura 2). La selección de díadas con una frecuencia de dos o más pases reduce el conjunto a 4 actores fundamentales en esta vertiente del juego. Assunçao apoya el juego en dos defensas preferentemente Juanito-, mientras que el pivote más habitual consiste en la tríada Assunçao-Luis Fernández-Arzu.

Figura 2. Red de pases de adaptación al juego del Real Betis Balompié.

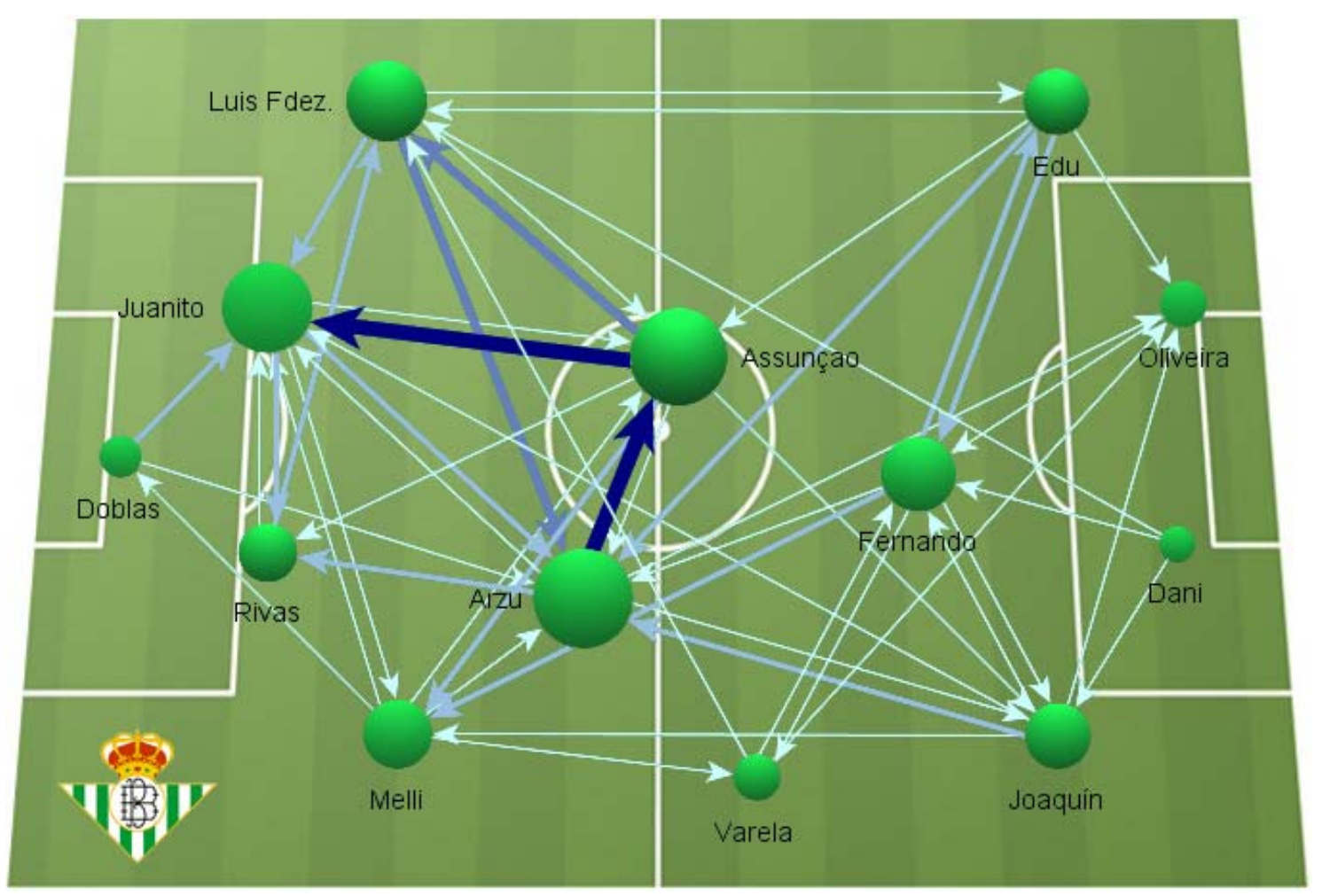

En segundo lugar, la matriz de los pases de juego revela con claridad la estrategia de ataque (Figura 3). La estructura sigue un patrón en diagonal hacia la derecha muy definido, buscando a Joaquín (y la conexión de éste con Oliveira). Otras alternativas en ataque pasan por Assunçao, Fernando y Edú. Sin embargo, hasta 4 líneas de pase preferenciales hacen de Joaquín el actor con mayor centralidad. Se 
trata de un estilo de juego directo, que sube de la defensa a la delantera pasando por el extremo derecho.

En contrapartida, la vulnerabilidad de esta apuesta de juego está en el elevado nivel de intermediación concentrado en un solo jugador. El marcaje directo a Joaquín y evitar que lleguen pases de balón al extremo son dos vías preferentes para contrarrestar este estilo de juego.

Figura 3. Red de pases de juego del Real Betis Balompié.

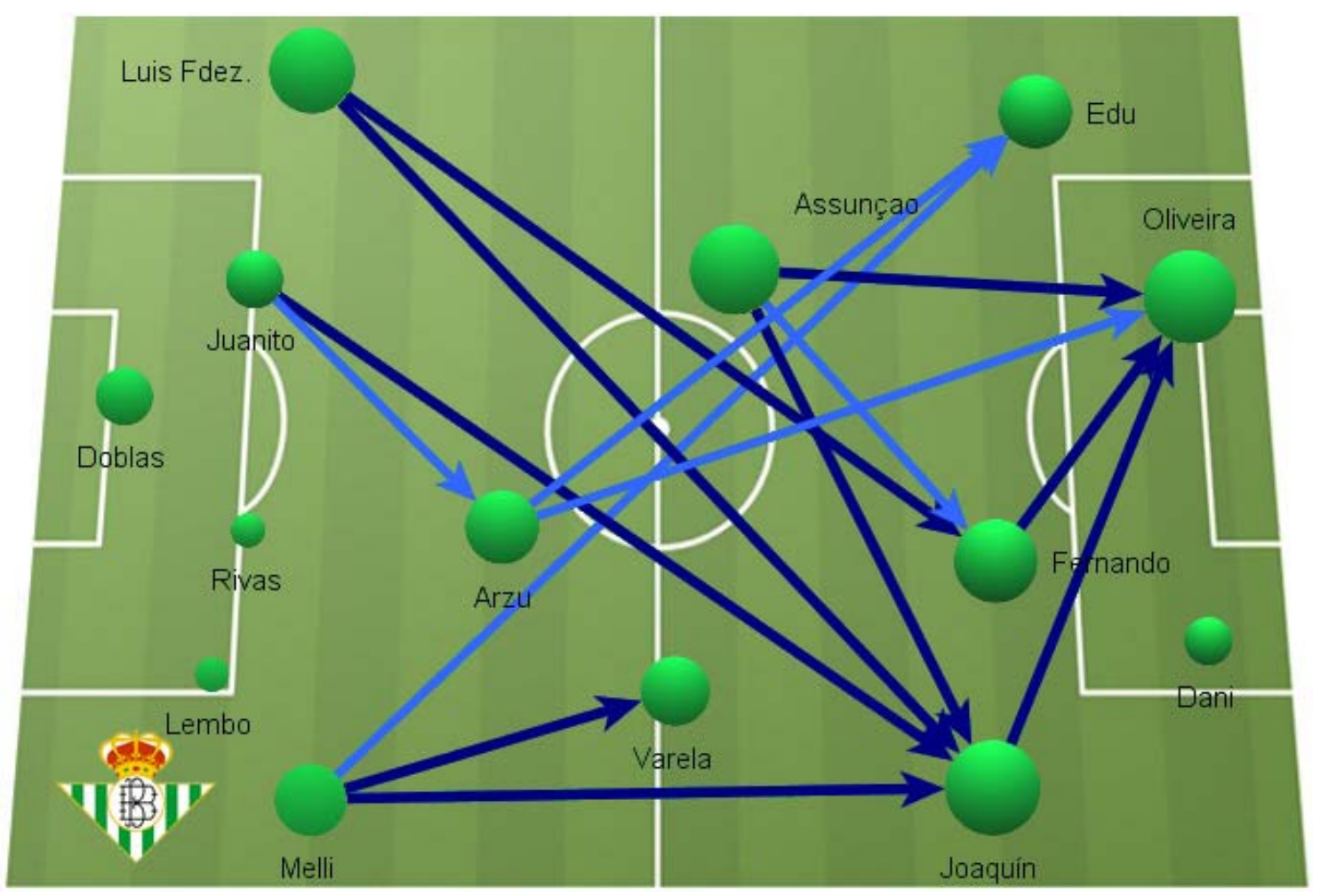

En definitiva vemos cómo el procesamiento de las matrices en función de los tipos de pase permite descubrir dinámicas de juego y adaptación al juego con mayor precisión ${ }^{3}$. En este trabajo pretendemos validar este examen exploratorio con un contraste específico. Concretamente, evaluaremos el carácter discriminante de las redes de juego y las redes de adaptación en los indicadores de centralidad individuales. Complementariamente, examinaremos la contribución del análisis de redes a la comprensión de las estadísticas individuales habituales.

\footnotetext{
${ }^{3}$ La combinación del análisis de redes de pases con la evaluación de elementos tácticos del partido se propuso inicialmente en Maya-Jariego \& Bohórquez (2011), donde se detallan las estrategias de codificación y análisis de datos. En cualquier caso, la metodología es en general la misma que se utiliza en el segundo partido analizado y que se describe a continuación.
} 


\section{Método}

Se analizó el partido de fútbol de final de Copa de S. M. El Rey disputado entre el Sevilla FC y el Getafe CF el 23 de junio de 2007. De este partido, se codificaron los 511 pases de balón efectuados entre los integrantes de cada uno de los equipos presentes en el terreno de juego (incluyendo a aquellos jugadores que accedieron como suplentes). Para tener una visión completa de las redes de pases es necesario incluir a todos aquellos que participaron en el partido, con independencia del número de minutos jugados (Bundio \& Conde, 2007).

La recolección de las redes de pases se complementaron con el análisis táctico del partido, realizada por un experto con larga experiencia profesional en la observación de sistemas de juego.

\section{Instrumentos}

Para la recogida de estadísticas tradicionales de frecuencias de acciones se usó una planilla de observación que incluye diferentes categorías: recuperaciones (por anticipación, físicas o por colocación), pérdidas (por control, por pase, por regate o por conducción), despejes (orientados o no orientados), centros al área (con o sin finalización), incursiones al área, tiros a puerta (entre los tres palos o fuera), faltas (cometidas o recibidas y sus sanciones asociadas), asistencias de gol y gol. La elección y definición operacional de las conductas definidas en dicha planilla fue sometida a juicio de expertos en el que participaron 5 entrenadores de fútbol de categoría nacional y 3 psicólogos expertos en psicología aplicada al deporte.

El instrumento de recogida de datos para la creación y análisis de las redes fue una matriz de doble entrada asimétrica en la que se codificaron los pases de balón entre los integrantes de un mismo equipo. Se utilizaron diferentes matrices para los pases recogidos en la categoría juego y aquellos referentes a la adaptación al juego. Además, los cambios de jugador en el terreno de juego implicaron cambios en la matriz de codificación, resultando en un total de 14 entradas por equipo. Un ejemplo de matriz se presenta en la Tabla 1, conteniendo los datos de la red total de pases del Sevilla FC. Se trata de una red valorada que permite evaluar la fuerza de las relaciones (la frecuencia de pases entre díadas) en diferentes umbrales de intensidad. 
Tabla 1. Matriz de codificación de pases.

\begin{tabular}{|c|c|c|c|c|c|c|c|c|c|c|c|c|c|c|}
\hline & $\frac{}{\frac{0}{\pi}}$ & 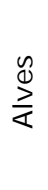 & 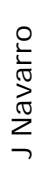 & $\begin{array}{l}\frac{0}{0} \\
\frac{0}{2} \\
\text { 山n }\end{array}$ & 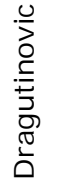 & $\frac{n}{\pi}$ & $\begin{array}{l}\frac{\nwarrow}{d} \\
\frac{\omega}{3} \\
\stackrel{0}{\Omega}\end{array}$ & 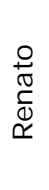 & $\begin{array}{l}\frac{\pi}{2} \\
\frac{1}{2} \\
\frac{2}{2}\end{array}$ & $\begin{array}{l}\stackrel{8}{\frac{0}{0}} \\
\frac{.0}{0} \\
\frac{\pi}{\leftarrow}\end{array}$ & 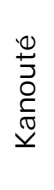 & 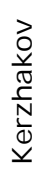 & $\begin{array}{l}\frac{\pi}{0} \\
\frac{0}{0} \\
0\end{array}$ & $\frac{\sqrt[T]{\frac{\pi}{\pi}}}{\sum}$ \\
\hline Palop & $*$ & 7 & 2 & 1 & 0 & 0 & 0 & 2 & 1 & 0 & 6 & 1 & 1 & 0 \\
\hline Alves & 0 & $*$ & 1 & 0 & 2 & 6 & 2 & 7 & 1 & 1 & 8 & 1 & 0 & 0 \\
\hline Navarro & 2 & 2 & $*$ & 1 & 0 & 0 & 2 & 0 & 2 & 0 & 0 & 0 & 0 & 0 \\
\hline Escudé & 4 & 0 & 0 & * & 5 & 1 & 1 & 3 & 5 & 1 & 1 & 2 & 0 & 0 \\
\hline Drago & 0 & 0 & 0 & 1 & $*$ & 1 & 1 & 8 & 10 & 2 & 8 & 0 & 2 & 0 \\
\hline Navas & 0 & 3 & 1 & 0 & 0 & $*$ & 0 & 1 & 1 & 0 & 1 & 0 & 0 & 0 \\
\hline Poulsen & 2 & 2 & 1 & 3 & 3 & 0 & * & 3 & 1 & 0 & 3 & 1 & 1 & 0 \\
\hline Renato & 0 & 5 & 0 & 3 & 2 & 2 & 2 & * & 7 & 2 & 2 & 1 & 0 & 0 \\
\hline Puerta & 0 & 1 & 0 & 1 & 4 & 1 & 2 & 2 & $*$ & 2 & 5 & 0 & 0 & 0 \\
\hline Fabiano & 0 & 0 & 0 & 0 & 1 & 0 & 1 & 1 & 0 & $*$ & 2 & 0 & 0 & 0 \\
\hline Kanouté & 0 & 4 & 0 & 0 & 2 & 4 & 2 & 2 & 3 & 2 & * & 5 & 1 & 0 \\
\hline Kerzha. & 0 & 0 & 0 & 0 & 0 & 0 & 0 & 1 & 2 & 0 & 3 & $*$ & 3 & 0 \\
\hline Duda & 0 & 0 & 0 & 0 & 0 & 0 & 0 & 0 & 0 & 0 & 0 & 0 & * & 1 \\
\hline Martí & 0 & 0 & 0 & 0 & 0 & 0 & 0 & 0 & 0 & 0 & 0 & 0 & 1 & * \\
\hline
\end{tabular}

\section{Procedimiento y análisis de datos}

La codificación de los datos se realizó a partir de la grabación en DVD del partido facilitada por el Servicio de Prensa del Sevilla FC. La imagen registra la evolución espacial del juego siguiendo en todo momento al jugador en posesión del balón y su actuación sobre el mismo. Así, la metodología de trabajo ha sido la observación sistematizada no participante en ambiente natural. Todas las codificaciones fueron realizadas por el mismo observador, con formación de entrenamiento en fútbol y experiencia en la consultoría a cuerpos técnicos de equipos profesionales.

Con el objetivo de asegurar la fiabilidad de los datos recogidos por este investigador, se sometió un cuarto del total de tiempo codificado ( 22.5 minutos) a registro observacional por parte de otro observador entrenado en la categorización propuesta, obteniéndose de la comparación inter-jueces un coeficiente Kappa de Cohen $=.852$, que asegura la fiabilidad de los datos. 
Para el tratamiento de los datos del partido se usaron Hoja de Cálculo Microsoft EXCEL en la ordenación de los datos relativos a la estadística tradicional y el software UCINET versión 6.0 (Borgatti, Everett \& Freeman, 2002) para el análisis de redes sociales. Una descripción de los indicadores de centralidad utilizados, así como de las estrategias de análisis y visualización de redes que aplicamos a continuación, puede consultarse en Molina, Quiroga, Martí, Maya-Jariego y de Federico (2006). Para el análisis estadístico de los indicadores obtenidos con el software UCINET se utilizó posteriormente el SPSS.17.0.

\section{Resultados}

La elaboración de los mapas de redes sociales de juego y adaptación al juego supuso la codificación de un total de 511 pases de balón (Tabla 2).

Tabla 2. Distribución de los pases de balón durante el encuentro.

\begin{tabular}{lccc} 
& Pases de juego & Pases de adaptación al juego & Total de pases \\
\hline Sevilla FC & $181(78.69 \%)$ & $49(21.30 \%)$ & 230 \\
Getafe CF & $207(73.66 \%)$ & $74(26.33 \%)$ & 281 \\
\hline
\end{tabular}

De estos pases se generó un mapa de redes sociales general para cada uno de los equipos que se presenta de forma comparativa en la Figura 4. La posición de los nodos que representan a los jugadores recoge la distribución espacial habitual de éstos en el campo de juego, mientras que los lazos que representan los pases de balón recogen la circulación de balón entre los diferentes jugadores, contemplándose la fortaleza del lazo en un mayor grosor de línea. El tamaño del nodo representa el grado de intermediación (betweeness). Se destacan con menor saturación de color a los suplentes que entraron para sustituir a un compañero a lo largo del partido.

El análisis de los lazos de la red del Sevilla FC coincide con una estrategia muy definida de ataque en largo, partiendo desde Palop hasta la recepción de Kanouté, pasando en ocasiones por Alves o Renato. También el desarrollo del juego vertical en bandas parece una característica del juego del equipo andaluz. La red del Getafe CF por contra, muestra un juego tendente a la utilización del carril central del campo, partiendo de Luis García hasta Belenguer y viceversa, pasando el balón directa o indirectamente- por Celestini y Casquero como medio de avance y/o retroceso. 
Figura 4. Red completa de pases del Sevilla FC.

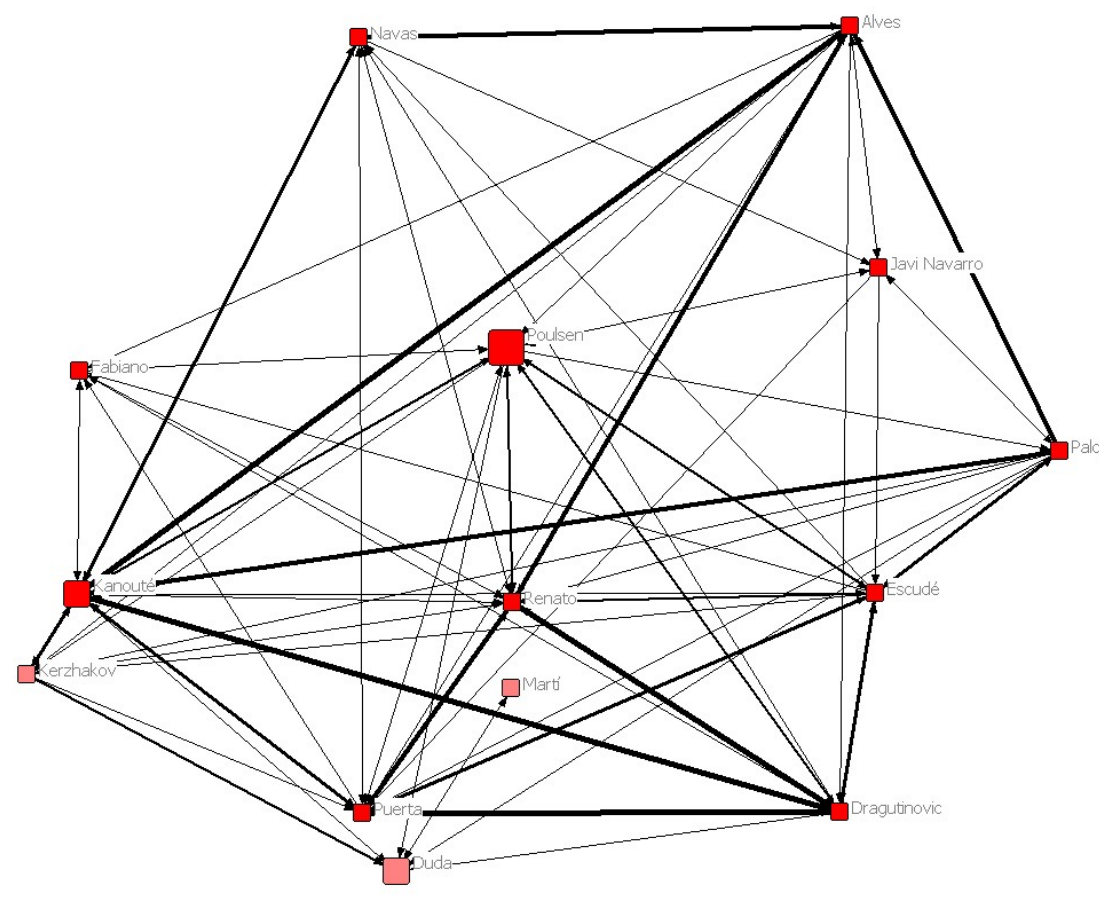

Figura 5. Red completa de pases del Getafe CF.

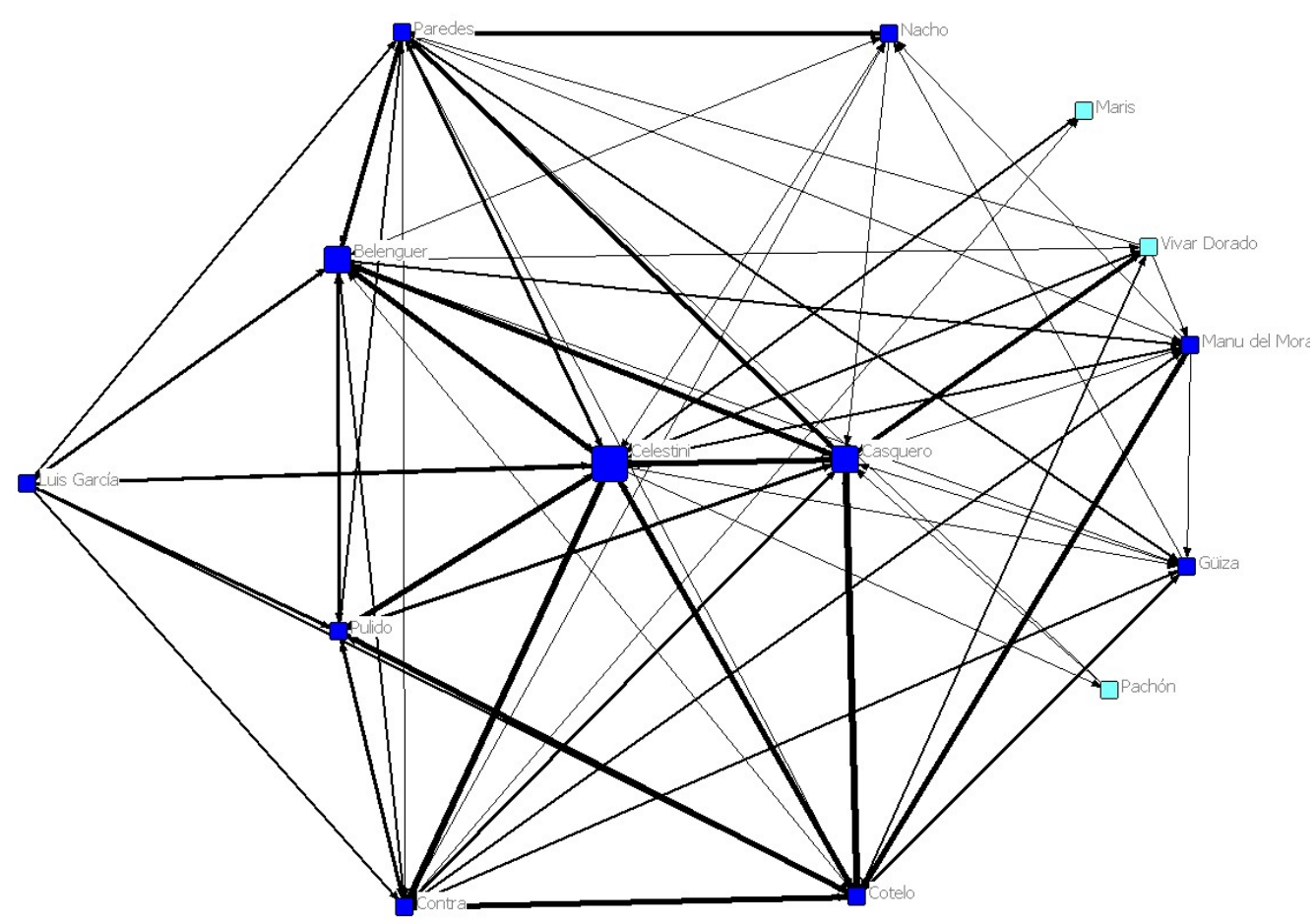


Tabla 3. Análisis de la centralidad de los principales nodos en las redes sociales del Sevilla FC y el Getafe CF.

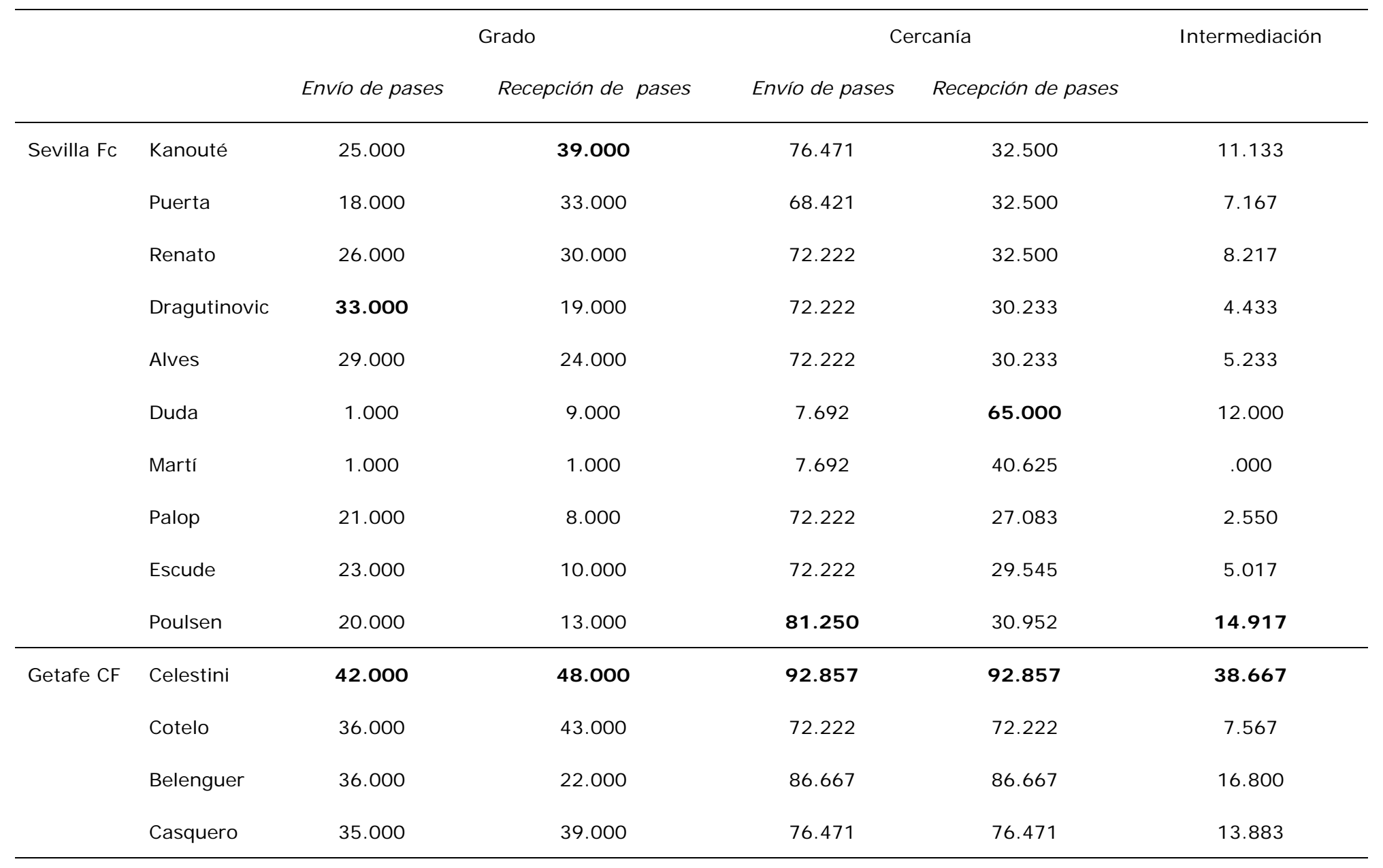


En la Tabla 3 se destacan los jugadores con mayor peso nodal. Los indicadores de centralidad muestran a Kanouté como el jugador con más oportunidades de recibir pases - Grado de entrada- y a Dragutinovic como el jugador que otorga el balón eficazmente en más ocasiones - Grado de salida- en el Sevilla FC. En lo referente a la Intermediación o grado en que un jugador realiza funciones de nexo en los caminos más cortos entre otros dos, Poulsen es el jugador de referencia del equipo andaluz. Respecto a la capacidad de resultar accesible de forma directa - Cercanía de entrada- Duda es el jugador de referencia, y Poulsen en la capacidad de acceder de forma directa a los compañeros - Cercanía de salida-. En lo referente al Getafe $\mathrm{CF}$, todos estos roles los ostenta Celestini, convirtiéndose también en jugador destacado en Cercanía de entrada y de salida Casquero.

El análisis diferencial de los distintos tipos de pases, de juego y de adaptación al juego, arroja mapas de redes diferentes que podemos ilustrar centrándonos en los grafos del Sevilla FC (Figura 6). En todos los casos el tamaño del nodo representa el grado de intermediación.

Figura 6. Redes de juego (izquierda) y adaptación al juego (derecha) del Sevilla FC.
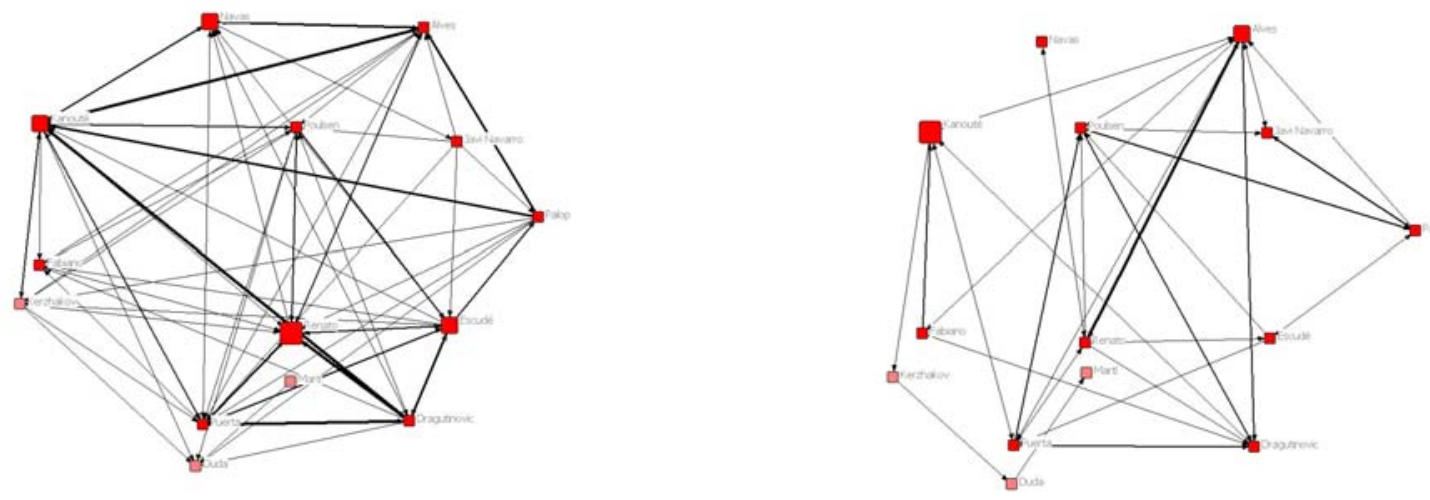

Un primer acercamiento revela diferencias evidentes en la densidad de ambas redes. La red de juego se caracteriza por una alta densidad con líneas preferentes de pase vertical por bandas y en largo para iniciar el juego del equipo en la punta de ataque (sustentada por la intermediación de Renato). El mapeo de la red de adaptación al juego resulta considerablemente menos denso, centrándose en el pivote de Renato y la movilidad de Alves para generar espacios entre los que poder distribuir el juego. El valor ponderado de densidad fue 3.6 veces mayor en los pases de juego que en los pases de adaptación. 
El análisis de los datos de centralidad de ambas redes (Tabla 4 para los datos de los jugadores con mayor peso nodal) permite discriminar ambas configuraciones mediante los cambios en los roles de los componentes del equipo. Mientras que en la red general y en la de juego la mayor oportunidad de recibir pases - Grado de entrada- es para Kanouté, en las situaciones en las que se busca aliviar la presión ejercida por el rival o generar movimiento en sus líneas, el protagonismo recae sobre Dragutinovic. Del mismo modo, la oportunidad de dar pases de forma directa e indirecta - Grado de salida- señalaba a Dragutinovic como jugador de referencia en las redes generales y de juego, adquiriendo ese rol Alves en la red de adaptación al juego. El peso del juego en la distribución de pases directos a otros jugadores - Cercanía de entrada- recae en Duda (jugador suplente) tanto en la red general como en la de juego. En la red general Poulsen mostraba la mayor accesibilidad en la generación de pases directos - Cercanía de salida-, mientras que la ostenta Renato en la red de juego; frente a Alves, Puerta y Renato en la red de adaptación al juego. Finalmente, en cuanto a la intermediación entre los diferentes nodos de la red, Renato en la red de juego y Kanouté en la red de adaptación al juego sustituyen a Poulsen, quien conectaba a los jugadores en el análisis general.

Tabla 4. Datos de centralidad del Sevilla FC en sus redes de juego y adaptación.

\begin{tabular}{|c|c|c|c|c|c|c|}
\hline & & \multicolumn{2}{|c|}{ Grado } & \multicolumn{2}{|c|}{ Cercanía } & \multirow[t]{2}{*}{ Intermediación } \\
\hline & & $\begin{array}{l}\text { Envío } \\
\text { de } \\
\text { pases }\end{array}$ & $\begin{array}{l}\text { Recepción } \\
\text { de pases }\end{array}$ & $\begin{array}{l}\text { Envío de } \\
\text { pases }\end{array}$ & $\begin{array}{l}\text { Recepción de } \\
\text { pases }\end{array}$ & \\
\hline \multirow{4}{*}{$\begin{array}{l}\text { Red de } \\
\text { juego }\end{array}$} & Dragutinovic & 30.000 & 11.000 & 43.333 & 28.889 & 2.400 \\
\hline & Kanouté & 22.000 & 35.000 & 43.333 & 31.707 & 10.300 \\
\hline & Duda & .000 & 8.000 & 7.143 & 65.000 & .000 \\
\hline & Renato & 20.000 & 24.000 & 44.828 & 31.707 & 14.250 \\
\hline \multirow{6}{*}{$\begin{array}{l}\text { Red de } \\
\text { adaptación } \\
\text { al juego }\end{array}$} & Alves & 10.000 & 5.000 & 50.000 & 22.807 & 36.000 \\
\hline & Dragutinovic & 3.000 & 8.000 & 40.625 & 22.807 & 14.250 \\
\hline & Martí & .000 & 1.000 & 7.143 & 23.000 & .000 \\
\hline & Puerta & 6.000 & 6.000 & 50.000 & 22.807 & 26.667 \\
\hline & Renato & 6.000 & 6.000 & 50.000 & 21.667 & 26.667 \\
\hline & Kanouté & 3.000 & 4.000 & 48.148 & 20.968 & 39.083 \\
\hline
\end{tabular}

Estos cambios en la centralidad de los diferentes nodos de las redes de juego y adaptación al juego, tanto entre sí como en referencia a la red general de pases, 
permite verificar la existencia de diferentes configuraciones tácticas en el juego y por lo tanto la relevancia de dicha diferenciación. Para asegurar que estas diferencias eran significativas y no un artefacto de medición, se sometieron los datos a la prueba no paramétrica para muestras independientes $U$ de MannWhitney. Se obtienen diferencias significativas en las comparaciones referidas a Centralidad en la recepción de pases $(Z=-3.792$; sig. $=.002)$, Centralidad en el envío de pases $(Z=-3.140$, sig. $=.000)$, Cercanía en la recepción de pases $(Z=-$ 3.227 , sig $=.000)$ y Cercanía para el envío de pases $(Z=-3.150$, sig. $=.000)$. Los datos relativos a la intermediación no mostraron diferencias estadísticamente significativas $(Z=-1.265$, sig. $>.05)$.

El análisis tradicional de un partido suele basarse en el recuento de frecuencias de acciones de corte técnico, tales como recuperaciones y pérdidas de balón, despejes y tiros a puerta, entre otros (Tabla 5). Las estadísticas individuales de juego pueden reinterpretarse tomando como referencia las redes de pases. Por ejemplo, la Figura 7 ilustra los datos de acciones individuales de Kanouté.

Tabla 5. Frecuencias de las categorías individuales de análisis del conjunto del Sevilla FC.

\begin{tabular}{|c|c|c|c|c|c|}
\hline \multicolumn{2}{|c|}{ Acciones tácticas } & \multirow{2}{*}{$\frac{F}{70}$} & \multicolumn{2}{|c|}{ Acciones tácticas } & \multirow{2}{*}{$\frac{F}{2}$} \\
\hline Recuperaciones & Anticipación & & Goles & Ocasión & \\
\hline & Colocación & 11 & & Gol & 1 \\
\hline & Física & 9 & & Asistencia & 0 \\
\hline \multirow[t]{4}{*}{ Pérdidas } & Control & 21 & Sanciones & Faltas cometidas & 16 \\
\hline & Conducción & 14 & & Faltas recibidas & 19 \\
\hline & Pase & 27 & & Tarjeta amarilla & 2 \\
\hline & Regate & 1 & & Tarjeta roja & 1 \\
\hline \multirow[t]{3}{*}{ Tiros a puerta } & Entre los palos & 6 & Centros al área & Finalizados & 9 \\
\hline & Fuera & 8 & & No finalizados & 17 \\
\hline & & & & Incursiones & 5 \\
\hline \multirow[t]{2}{*}{ Despejes } & Orientado & 33 & & & \\
\hline & No orientado & 8 & & & \\
\hline
\end{tabular}

Kanouté es el jugador con mayor centralidad en la recepción de pases de juego. Los registros individuales muestran un elevado número de pérdidas de balón. Podemos contextualizar este dato si tenemos en cuenta que sus líneas de pase parten de Palop y Alves, jugadores pertenecientes a la defensa del equipo. Por tanto, gran 
parte del juego directo que recibe es aéreo. Atendiendo al tipo de pérdidas de balón, el $55.5 \%$ se producen por errores en el control del balón y el $22.2 \%$ en el pase del esférico. Las dificultades en el control de pases aéreos se corresponden con la configuración táctica reflejada en la red de pases. También nos ayuda a entender por qué se ve envuelto en un gran número de faltas, como muestran los datos tanto de realización como de recepción de faltas. El rol de recepción de pases de Kanouté se enfrenta con el rol defensivo ejercido por Belenguer, vértice clave en la red de oposición del Getafe CF.

Veamos también el caso de Poulsen, que ocupa el centro del campo. El número de recuperaciones de balón de este jugador ( 7 en total) en conjunción con el limitado número de pérdidas de posesión ( 3 durante el total del encuentro) guarda relación con su rol en la red de pases. Recordemos que los datos de centralidad lo señalan como el jugador con mayor proximidad al resto de compañeros, especialmente en la generación de pases según el indicador de cercanía de salida. También es una pieza clave en la intermediación entre los diferentes futbolistas. Como vemos con ambos ejemplos, la estructura que sustenta la interacción, la red social del equipo, se corresponde con la conducta deportiva de los jugadores a nivel individual.

Figura 7. Registro de acciones individuales de Kanouté (Sevilla FC) por cuartos de partido.
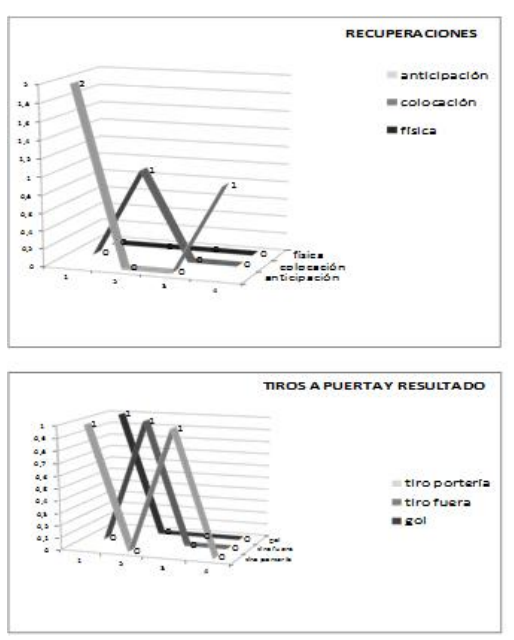
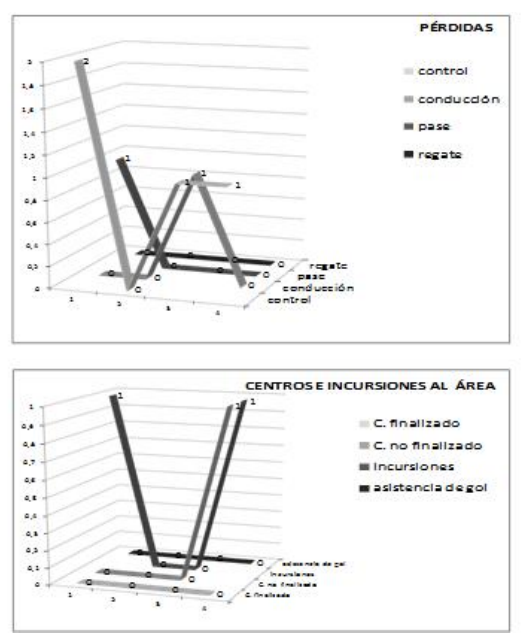

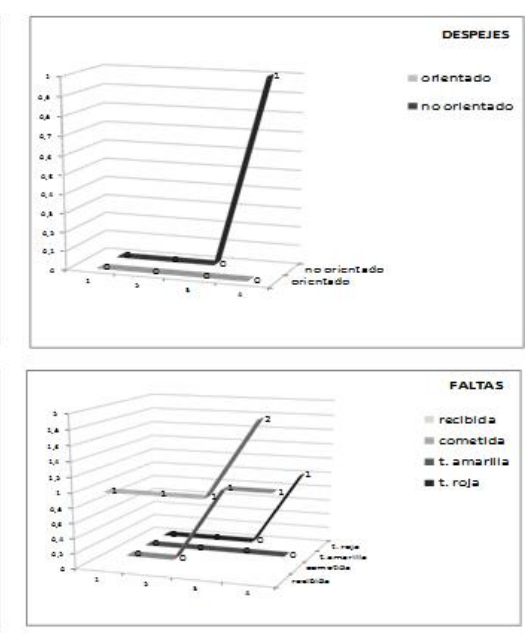

\section{Discusión}

La representación en un mismo mapa del total de relaciones establecidas entre los jugadores de un equipo permite identificar patrones de juego (Lago \& Anguera, 2003). Desde ese punto de vista, el equipo es cualitativamente diferente de la suma de las individualidades y sus contribuciones (Franzoi, 2007; Olmedilla, Ortega, Almeida, Lameira, Villalonga, Sousa, Torregrosa, Cruz, García-Mas, 2011), 
acercándonos a una mejor comprensión del trabajo grupal. El análisis de redes sociales aplicado al fútbol sirve para representar el trabajo colectivo. Al mismo tiempo, permite analizar el papel que los diferentes integrantes de la red tienen en el funcionamiento de la misma, contemplando así tanto análisis macro-micro como micro-micro y micro-macro. La posibilidad de re-interpretar los datos estadísticos individuales basándonos en una comprensión de la dinámica grupal - por extensión, los aspectos tácticos del juego- es un ejemplo de cómo la conciliación de los indicadores tradicionales con los más novedosos - en este caso, las redes de pases-, permite abarcar todos los niveles de análisis.

El mapeo de las redes sociales de los equipos participantes en un encuentro posibilita el examen en paralelo de las configuraciones tácticas de los mismos, contribuyendo a la comprensión del juego colectivo como resultado simultáneamente de la cooperación intra-grupal y la oposición intergrupal (Riera, 1985). Por ejemplo, el estudio simultáneo de las redes del Sevilla FC y el Getafe CF permite formular la hipótesis de que la utilización de las bandas por parte del equipo andaluz y la ocupación del carril central del juego del equipo madrileño se deben a la búsqueda estratégica del espacio por parte de los contendientes. Sería necesario ahondar en esta hipótesis mediante el análisis temporal y/o longitudinal del juego para dilucidar la medida en que las configuraciones tácticas desarrolladas durante el partido se deben a las relaciones de oposición establecidas con los diferentes contrincantes (Cotta et al., 2011) o a un esquema de juego estable y preestablecido por parte de los equipos.

Abordar el análisis de dos tipos de redes diferentes, supone aplicar un filtro cuyos productos de análisis capacitan para una mejor comprensión de los aspectos tácticos en sus diferentes facetas. Anteriormente Chicote et al. (2009) y Morante (2009) ya sugerían la utilidad del empleo de filtros para la separación comprensiva de elementos tácticos y estratégicos. En esto radica la principal aportación de este estudio: en la propuesta de un procedimiento para distinguir entre estrategias de juego y adaptación en las redes de pases. Tanto el análisis descriptivo como las comparaciones no paramétricas confirman la existencia de dos redes diferenciadas. Ambas se corresponden además con la interpretación cualitativa del juego y con las estadísticas individuales. Aunque estudios anteriores ya abogaban por la separación de pases de balón en función de su intención en el juego (da Costa et al. 2011), los autores llevaban a cabo una división en fases ofensiva y defensiva del juego, mientras que nuestra aportación pretende incidir en el significado comunicativo de los pases de balón en las diferentes redes mapeadas. Además, la utilización del análisis de redes permite la obtención de indicadores de trabajo colectivo así como de los roles de cada jugador en función de la vertiente del juego analizada, 
aspectos que no se trataron en el estudio de da Costa et al. (2011). Futuros trabajos podrían combinar diferentes categorizaciones tácticas del juego con el uso de las técnicas de análisis de redes, evidenciando así la versatilidad y sensibilidad de la herramienta presentada en el análisis táctico del comportamiento deportivo grupal. Se trata de estrategias de investigación acordes con un enfoque ecológico y multidimensional en el estudio de la práctica deportiva (Ruiz, Sánchez, Durán, Jiménez, 2006).

La red de adaptación representa las estrategias que pretenden evitar la posesión del balón por parte del contrario. Se trata de uno de los factores determinantes del resultado del juego (Lago \& Martín, 2007). Resulta de gran interés evaluar en futuros estudios qué propiedades de la red se relacionan con la resistencia a la interceptación de pases. Es probable que dichos indicadores tengan gran variabilidad entre partidos, dependiendo de los contrincantes. También podrían ser más elevados en los encuentros con más contención en el juego, como puede ser el caso de las finales de Copa analizadas.

Por su parte, la efectividad de la red de juego parece relacionarse con la existencia de un núcleo bien coordinado de jugadores. Estudios anteriores han encontrado un mejor rendimiento en las redes de pases de centralización reducida, con elevada transitividad y que aumentan en el nivel de aglomeración a lo largo del partido (Bundio \& Conde, 2007; Cotta et al., 2011; Lee et al., 2005; Onody \& de Castro, 2004). En cualquier caso, para evaluar el rendimiento de redes específicas parece necesario comparar la estructura de pases con la del oponente. De ese modo se puede valorar la confrontación de las diferentes configuraciones tácticas.

El estudio de las redes de pases también se ha mostrado efectivo en el nivel individual, caracterizando el rendimiento y el papel desempeñado por cada jugador. Por ejemplo, el grado de intermediación puede ayudar a determinar los ejes en la distribución del balón (Cotta et al., 2011). Hemos intentado mostrar que la combinación de los patrones de juego y adaptación enriquece el análisis individual, aportando una visión más compleja de las diferentes facetas del juego. En el caso del Real Betis Balompié, Assunçao tiene un papel destacado en ambas redes, resultando por tanto clave en la articulación táctica del equipo. Por lo que corresponde a los actores, sería de interés investigar la correlación entre los indicadores de centralidad y las estadísticas individuales tradicionales.

Los datos de nuestro estudio se limitan a dos partidos concretos. Es necesario contrastar estas observaciones en contextos diferentes. No obstante, la originalidad de la propuesta está en la oportunidad de combinar el análisis de redes con el examen táctico. Hemos propuesto un nuevo enfoque en el tratamiento de los datos 
de distribución del juego. Esta perspectiva resulta prometedora tanto para la investigación como en el contexto del entrenamiento deportivo. El estudio de las redes de pases no va a impedir que leamos crónicas de fútbol en las que el resultado no se corresponde con las características del juego de los contendientes. Cualquier lector de prensa deportiva sabe que los palos, "la suerte" y los árbitros también juegan. Con este estudio hemos intentado poner de manifiesto que el análisis empírico de la estructura de pases de cada equipo puede incorporarse entre las herramientas que contribuyen a una mejor comprensión del juego y, de un modo práctico, al adiestramiento táctico en fútbol.

\section{Referencias bibliográficas}

Anguera, M. T.; Blanco, Á.; Losada, J. L. y Hernández-Mendo, A. (2000). La metodología observacional en el deporte: conceptos básicos. Lecturas: EF y Deportes. Revista Digital, 24. [http://www.efdeportes.com/efd24b/obs.htm [Consulta: 19 noviembre 2011].

ARSFútbol (2011). Manchester United Team Review: la construcción de vínculos como eje del juego en el equipo. Informe de resultados de la Semifinal UEFA Champions 2011. Disponible en: http://arsfutbol.wordpress.com

Borgatti, S.P., Everett, M.G. y Freeman, L.C. (2002). Ucinet for Windows: Software for Social Network Analysis. Harvard, MA: Analytic Technologies.

Bundio, J. y Conde, M. (2007). Exploraciones en Fútbol y Redes Sociales. Análisis del desempeño deportivo durante la Eurocopa 2004 a partir del análisis de redes sociales. REDES, Revista Hispana para el Análisis de Redes Sociales, $13(2)$.

Castellano, J.; Hernández-Mendo, A. (2002). Análisis diacrónico de la acción de juego en fútbol. Lecturas: EF y Deportes. Revista Digital, 49. http://www.efdeportes.com/efd49/diacro.htm [Consulta: 24 noviembre 2011]

Castellano, J., Hernández-Mendo, A. y Haro, J. A. (2002). Mapas socioconductuales de la selección francesa en el mundial de fútbol de Francia '98. Revista de Psicología del Deporte, 11(1) 35-51.

Chicote, J.; Morante, J. C. y Vaquera, A. (2009). Propuesta metodológica para el análisis táctico de las acciones ofensivas en equipos profesionales de baloncesto. Revista de Psicología del Deporte, 9 (Suplemento), 75.

Cotta, C. Mora, A. M., Merelo-Molina, C. y Merelo, J. J. (2011). Fifa World Cup 2010: A Network Analysis of the Champion Team Play. arXiv:1108.0261v1 
Da Costa, I. T.; Garganta, J.; Greco, P. J. \& Mesquita, I. (2011). Proposta de avaliação do comportamento tático de jogadores de Futebol baseada em princípios fundamentais do jogo. Motriz, Rio Claro, 17(3), 511-524.

Franzoi, S. L. (2007). Psicología Social (4ạ ed.). D. F.: México.

Galeote, F. (2003). Análisis táctico del juego en función del puesto. Apuntes del Curso Oficial de Entrenadores de Fútbol del Centro de Estudios, Desarrollo e Investigación del Fútbol Andaluz.

Gould, P., and Gatrell, A. (1979). A structural analysis of a game: The Liverpool v. Manchester United Cup final of 1977. Social Networks 2, 253-273.

Hernández-Mendo, A. y Canto, J. (2003). El liderazgo en los grupos deportivos. En A. Hernández Mendo. Psicología del Deporte (Vol.1): Fundamentos 2 (pp. 628). Buenos Aires: Tulio Guterman.

Hernández-Mendo, A., González, S., Ortega, A., Ortega, J. y Rondan, R. (1999). La sociometría y los mapas conductuales en el baloncesto. Lecturas: EF y Deportes. Revista Digital, 16, octubre 1999 http://www.sirc.ca/revista/ [Consulta: 29 febrero 2012].

Klein, M. y Christiansen, G. (1969). Group composition, group structure and group effectiveness of basketball teams. In J. S. Loy y G. S. Kenyon (Eds.). Sport, Culture and Society. New York: McMillan.

Lacuesta Salazar, F. (1997) Tratado de Fútbol. Técnica, acciones del juego, estrategia y Táctica. Gymnos, Madrid.

Lago, C.; Anguera, M. T. (2003). Utilización del análisis secuencial en el estudio de las interacciones entre jugadores en el fútbol de rendimiento. Revista de Psicología del Deporte, 12(1), 27-37.

Lago, C. and Martín, R. (2007). Determinants of possession of the ball in soccer. J ournal of Sports Sciences, 25 (9), 969-974.

Lee, J., Borgatti, S. P., Molina, J. L. y Merelo, J. J. (2005). Who Passes To Whom: Analysis Of Optimal Network Structure In Soccer Matches. XXV International Sunbelt Social Network Conference, Redondo Beach, CA (EUA), February 19, 2005.

Maya J ariego, I. y Bohórquez, M. R. (2011). Alternativas a la estadísticas tradicional en deportes de equipo. Indicadores de trabajo colectivo. Taller del XIII Congreso Andaluz de Psicología de la Actividad Física y el Deporte. Facultad de Psicología de la Universidad de Sevilla, 16 a 19 de noviembre de 2011. 
Molina, J. L., Quiroga, A., Martí, J., Maya Jariego, I. \& de Federico (Eds.) (2006). Talleres de autoformación con programas informáticos de análisis de redes sociales. UAB: Barcelona.

Morante, J. C. (2009). Análisis del rendimiento en deportes de equipo: del registro estadístico al análisis táctico. Actas del II Congreso internacional de Deportes de Equipo. Coruña: Universidad de Coruña.

Moreno, M. (1998) Táctica y sistema de juego. Real Federación Española de Fútbol: Madrid.

Olmedilla, A., Ortega, E., Almeida, P., Lameira, J., Villalonga, T., Sousa, C., Torregrosa, M., Cruz, J. y García-Mas, A. (2011). Cohesión y cooperación en equipos deportivos. Anales de Psicología, 27 (1), 232-238.

Onody, R. y de Castro, P. (2004). Complex network study of brazilian soccer players. Physical Review E, 70 (3): 037103.

Peñaranda-Ortega, M., Quiñones-Vidal, E. y Osca-Lluch, J. (2009). La revista Anales de Psicología desde una perspectiva de redes sociales. Anales de Psicología, 25 (2), 199-208.

Riera, J. (1985). Introducción a la psicología del deporte. Barcelona: Martínez Roca.

Ruiz, L. M., Sánchez, M., Durán, J. y Jiménez, C. 2006. Los expertos en el deporte: su estudio y análisis desde una perspectiva psicológica. Anales de Psicología, 22 (1), 132-142.

Santoyo, C. (1994). Sociometría conductual: el diseño de mapas socioconductuales. Revista Mexicana de Análisis de la Conducta, 20 (2), 183-205.

Sautu, L. M., Garay, J. O. y Hernández-Mendo, A. (2009). Observación y análisis de las interacciones indirectas en el baloncesto ACB. Cuadernos de Psicología del Deporte, 9, 69.

Wasserman, S. and Faust, K. (1994). Social Network Analysis: Methods and Applications. Cambridge: Cambridge University Press. 